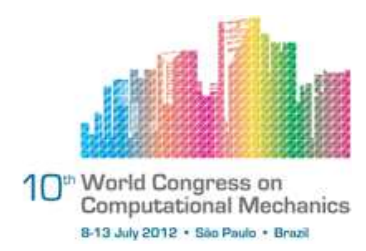

\title{
MATERIAL MODELLING OF EVOLVING ELASTIC AND PLASTIC ANISOTROPY WITH APPLICATION TO DEEP DRAWING PROCESSES
}

\author{
I. N. Vladimirov ${ }^{1}$, S. Reese ${ }^{1}$ \\ ${ }^{1}$ Institute of Applied Mechanics, RWTH Aachen University \\ (ivaylo.vladimirov@ rwth-aachen.de)
}

\begin{abstract}
The paper proposes a hyperelasticity-based concept of finite strain plasticity with combined hardening using evolving structure tensors to represent the evolution of elastic and plastic anisotropy in the material. By defining the Helmholtz free energy density and the yield surface as functions of the evolving structure tensors, we are able to describe both evolving elastic and plastic anisotropy, respectively. The model considers also nonlinear kinematic and isotropic hardening and is derived from a thermodynamic framework based on the multiplicative split of the deformation gradient. The kinematic hardening component represents a continuum extension of the classical rheological model of Armstrong-Frederick kinematic hardening. Exploiting the dissipation inequality leads to the important result that the model includes only symmetric tensor-valued internal variables. Evolution of elastic and plastic anisotropy is numerically investigated by means of simulations of cylindrical deep drawing of metal sheets and thermoforming of thermoplastic polymer blends.
\end{abstract}

Keywords: Evolving anisotropy, Structure tensors, Deep drawing.

\section{INTRODUCTION}

Finite element analysis is increasingly being used in modern sheet forming processes, provided that it relies on modules that capture the realistic behaviour sufficiently well. One of the important factors influencing the simulation result is the underlying material model. Due to the characteristic anisotropy of sheet metals, the material model should be capable of predicting initial and deformation-induced anisotropy. Sheet metals exhibit anisotropic plastic behaviour due to their orientation-dependent microstructure. Many problems in sheet metal forming processes arise due to the inherent sheet anisotropy. During the rolling process of the sheet, large plastic deformations occur which may induce texture and are responsible for the initial anisotropy. In addition, sheet metal parts are subjected to stretching, bending and reverse bending during forming, and an accurate prediction of e.g. the blank springback requires the use of an appropriate material model, which is capable of modelling the kinematic and isotropic hardening behaviour of metals.

Various approaches for introducing plastic anisotropy into the finite element analysis of sheet metal forming are popular nowadays. The initial plastic anisotropy can be incorpo- 
rated either through an anisotropic yield surface or directly by means of a crystallographic texture model. Here, one basically distinguishes between empirical and phenomenological anisotropic yield functions ([2], [1], [8]), where the anisotropy coefficients can be obtained from mechanical tests, and texture-based models ([3] the coefficients of which are directly determined based on experimentally obtained grain orientation distributions. Nowadays many scientists utilize the microstructural behaviour into their constitutive concept. Despite the significant progress achieved in material modelling at meso and micro scales, however, the methods are still computationally too expensive and time consuming. Approaches based on phenomenological continuum mechanics are frequently used due to their relatively low computational effort, which makes them especially advantageous for the simulation of real forming processes. Anisotropic continuum mechanical approaches in the regime of large deformations can be, in general, separated into two groups: additive formulations, either in the logarithmic strain space ([4]) or using generalized stress-strain measures ([5]), and multiplicative formulations utilizing the classical split of the deformation gradient ([7], [11]).

In this work, we discuss a finite strain continuum mechanical model combining both nonlinear isotropic hardening and nonlinear kinematic hardening. The kinematic hardening component represents a continuum extension of the classical Armstrong-Frederick concept based on a strain-like tensor-valued internal variable. The material model includes, in addition, evolving elastic and plastic anisotropy and can be understood as an extension of a recently published finite-strain framework for plastic anisotropy and combined hardening [11]. In the present model, the evolution of elastic anisotropy is represented by representing the Helmholtz free energy as a function of a family of evolving structure tensors. In addition, plastic anisotropy is modelled via the dependence of the yield surface on the plastic deformation and on the same family of structure tensors. Exploiting the dissipation inequality leads to the important result that all tensor-valued internal variables are symmetric. Thus, the integration of the evolution equations can be efficiently performed by means of a newly-developed form of the exponential map algorithm [9] based on an implicit time integration scheme. It automatically satisfies plastic incompressibility in every time step, and in addition, has the advantage of retaining the symmetry of the internal variables.

\section{MATERIAL MODEL}

The derivation of the constitutive model is based upon the multiplicative split of the deformation gradient $\mathbf{F}$ into elastic $\mathbf{F}_{e}$ and plastic $\mathbf{F}_{p}$ parts, where, in order to model kinematic hardening, the plastic deformation gradient is additionally decomposed into elastic $\mathbf{F}_{p_{e}}$ and inelastic $\mathbf{F}_{p_{i}}$ parts. The Helmholtz free energy is assumed to read

$$
\psi=\psi_{e}\left(\mathbf{C}_{e}, \mathbf{M}_{1}, \mathbf{M}_{2}\right)+\psi_{\text {kin }}\left(\mathbf{C}_{p_{e}}\right)+\psi_{\text {iso }}(\kappa)
$$

where $\psi_{e}$ is an isotropic function of the elastic right Cauchy-Green tensor $\mathbf{C}_{e}$ and two structure tensors $\mathbf{M}_{1}$ and $\mathbf{M}_{2}$. These second order structure tensors live as $\mathbf{C}_{e}$ in the intermediate configuration. The corresponding structure tensors in the reference configuration are defined as dyadic products of the preferred material orientations, i.e. principal axes of anisotropy:

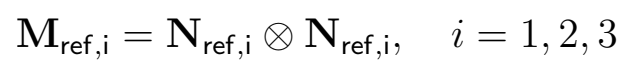


The unit vectors of the preferred material orientations in the reference configuration are assumed to be mapped to the intermediate configuration by means of the plastic deformation gradient $\mathbf{F}_{p}$ and are subsequently normalized (see [6]):

$$
\mathbf{N}_{\mathrm{i}}=\frac{\mathbf{F}_{p}\left[\mathbf{N}_{\text {ref }, \mathrm{i}}\right]}{\left\|\mathbf{F}_{p}\left[\mathbf{N}_{\text {ref }, \mathrm{i}}\right]\right\|}
$$

Exploiting the Clausius-Duhem form of the second law of thermodynamics yields the important result that the so-called Mandel-type stress tensors

$$
\boldsymbol{\Sigma}=2 \mathbf{C}_{e} \frac{\partial \psi_{e}}{\partial \mathbf{C}_{e}}-2 \sum_{i=1}^{2}\left(\frac{\partial \psi_{e}}{\partial \mathbf{M}_{\mathrm{i}}} \mathbf{M}_{\mathrm{i}}-\operatorname{tr}\left(\frac{\partial \psi_{e}}{\partial \mathbf{M}_{\mathrm{i}}} \mathbf{M}_{\mathrm{i}}\right) \mathbf{M}_{\mathrm{i}}\right)-2 \mathbf{F}_{p_{e}} \frac{\partial \psi_{\mathrm{kin}}}{\partial \mathbf{C}_{p_{e}}} \mathbf{F}_{p_{e}}^{T}
$$

and

$$
\boldsymbol{\Sigma}_{\text {kin }}=2 \mathbf{C}_{p_{e}} \frac{\partial \psi_{\text {kin }}}{\partial \mathbf{C}_{p_{e}}}
$$

are symmetric. A consequence of this latter symmetry is the fact that only the symmetric parts of the corresponding thermodynamically conjugate velocity gradients are relevant. This yields a framework with symmetric tensor-valued strain-like internal variables. The ClausiusDuhem inequality

$$
\left(\mathbf{S}-2 \mathbf{F}_{p}^{-1} \frac{\partial \psi_{e}}{\partial \mathbf{C}_{e}} \mathbf{F}_{p}^{-T}\right) \cdot \frac{1}{2} \dot{\mathbf{C}}+\boldsymbol{\Sigma} \cdot \mathbf{d}_{p}+\boldsymbol{\Sigma}_{\mathrm{kin}} \cdot \mathbf{d}_{p_{i}}+R \dot{\kappa}+\sum_{i=1}^{2} \boldsymbol{\Gamma}_{i} \cdot \dot{\mathbf{M}}_{\mathrm{ref}, \mathrm{i}} \geq 0
$$

is satisfied by a physical expression for the second Piola-Kirchhoff stress tensor

$$
\mathbf{S}=2 \mathbf{F}_{p}^{-1} \frac{\partial \psi_{e}}{\partial \mathbf{C}_{e}} \mathbf{F}_{p}^{-T}
$$

and evolution equations for the internal variables. Note that in this work no evolution equations for the structure tensors $\mathbf{M}_{\text {ref,i }}$ are proposed, thus the stress-like tensors $\boldsymbol{\Gamma}_{i}$ do not have to be computed. Clearly, experimental evidence of evolving anisotropy is required in order to define realistic evolution equations for the structure tensors as internal variables. Nevertheless, due to the dependence on the non-constant structure tensors $\mathbf{M}_{i}$, the effect of large plastic deformation on the evolution of elastic and plastic anisotropy is still taken into account in the current approach.

Due to numerical reasons the constitutive model is specified in the undeformed or reference configuration. After a pull-back to the reference configuration, the evolution equations for the internal variables of the model $\mathbf{C}_{p}$ (plastic deformation), $\mathbf{C}_{p_{i}}$ (kinematic hardening) and $\kappa$ (isotropic hardening) read

$$
\dot{\mathbf{C}}_{p}=2 \dot{\lambda} \frac{\overline{\mathbf{Y}} \mathbf{C}_{p}}{\sigma_{\text {Hill }}}, \quad \dot{\mathbf{C}}_{p_{i}}=2 \dot{\lambda} \frac{b}{c} \mathbf{Y}_{k i n}^{D} \mathbf{C}_{p_{i}}, \quad \dot{\kappa}=\sqrt{\frac{2}{3}} \dot{\lambda}
$$

Here, $\mathbf{Y}$ and $\mathbf{Y}_{k i n}$ are stress-like quantities resulting from the pull-back of the Mandel-type stress tensors $\Sigma$ and $\Sigma_{\text {kin }}$ into the reference configuration:

$$
\mathbf{Y}=\mathbf{C S}-\mathbf{C}_{p} \mathbf{X}+\mathbf{C}_{p} \mathbf{G}_{4}+\mathbf{C}_{p} \mathbf{G}_{6}, \quad \mathbf{Y}_{k i n}=\mathbf{C}_{p} \mathbf{X}
$$


The stress-like second-order tensors $\mathbf{G}_{4}$ and $\mathbf{G}_{6}$ are derived from the anisotropic Helmholtz energy part $\psi_{e_{a n}}$ :

$$
\mathbf{G}_{2 i+2}=\frac{2}{\mathbf{C}_{p} \cdot \mathbf{M}_{\mathrm{ref}, \mathrm{i}}} \frac{\partial \psi_{e_{a n}}}{\partial\left(\mathbf{C}_{e} \cdot \mathbf{M}_{\mathbf{i}}\right)}\left(-\mathbf{C}_{p}^{-1} \mathbf{C M}_{\mathrm{ref}, \mathrm{i}}+\frac{\operatorname{tr}\left(\mathbf{C} \mathbf{M}_{\mathrm{ref}, \mathrm{i}}\right)}{\mathbf{C}_{p} \cdot \mathbf{M}_{\mathrm{ref}, \mathrm{i}}}\right) \mathbf{M}_{\mathrm{ref}, \mathrm{i}} \quad i=1,2
$$

whereas $\overline{\mathbf{Y}}$ reads as follows:

$$
\begin{aligned}
\overline{\mathbf{Y}} & =m_{1} \mathbf{Y}^{D}+\left(l_{3} \frac{\operatorname{tr}\left(\mathbf{Y}^{D} \mathbf{C}_{p} \mathbf{M}_{\mathrm{ref}, 2}\right)}{\mathbf{C}_{p} \cdot \mathbf{M}_{\mathrm{ref}, 2}}-l_{2} \frac{\operatorname{tr}\left(\mathbf{Y}^{D} \mathbf{C}_{p} \mathbf{M}_{\mathrm{ref}, 1}\right)}{\mathbf{C}_{p} \cdot \mathbf{M}_{\mathrm{ref}, 1}}\right) \mathbf{1} \\
& +\left(l_{4} \frac{\operatorname{tr}\left(\mathbf{Y}^{D} \mathbf{C}_{p} \mathbf{M}_{\mathrm{ref}, 1}\right)}{\mathbf{C}_{p} \cdot \mathbf{M}_{\mathrm{ref}, 1}}+m_{2} \frac{\operatorname{tr}\left(\mathbf{Y}^{D} \mathbf{C}_{p} \mathbf{M}_{\mathrm{ref}, 2}\right)}{\mathbf{C}_{p} \cdot \mathbf{M}_{\mathrm{ref}, 2}}\right) \frac{\mathbf{C}_{p} \mathbf{M}_{\mathrm{ref}, 1}}{\mathbf{C}_{p} \cdot \mathbf{M}_{\mathrm{ref}, 1}} \\
& +\left(m_{2} \frac{\operatorname{tr}\left(\mathbf{Y}^{D} \mathbf{C}_{p} \mathbf{M}_{\mathrm{ref}, 1}\right)}{\mathbf{C}_{p} \cdot \mathbf{M}_{\mathrm{ref}, 1}}+m_{3} \frac{\operatorname{tr}\left(\mathbf{Y}^{D} \mathbf{C}_{p} \mathbf{M}_{\mathrm{ref}, 2}\right)}{\mathbf{C}_{p} \cdot \mathbf{M}_{\mathrm{ref}, 2}}\right) \frac{\mathbf{C}_{p} \mathbf{M}_{\mathrm{ref}, 2}}{\mathbf{C}_{p} \cdot \mathbf{M}_{\mathrm{ref}, 2}} \\
& +\left(\mathbf{C}_{p} \mathbf{M}_{\mathrm{ref}, 1} \mathbf{Y}^{D}+\mathbf{Y}^{D} \mathbf{C}_{p} \mathbf{M}_{\mathrm{ref}, 1}\right) \frac{l_{5}}{\mathbf{C}_{p} \cdot \mathbf{M}_{\mathrm{ref}, 1}} \\
& +\left(\mathbf{C}_{p} \mathbf{M}_{\mathrm{ref}, 2} \mathbf{Y}^{D}+\mathbf{Y}^{D} \mathbf{C}_{p} \mathbf{M}_{\mathrm{ref}, 2}\right) \frac{l_{6}}{\mathbf{C}_{p} \cdot \mathbf{M}_{\mathrm{ref}, 2}}
\end{aligned}
$$

Plastic anisotropy is included in the model by defining an explicit dependence of the yield function on the structure tensors. Here, the classical Hill anisotropic yield criterion of [2] has been used

$$
\Phi=\sigma_{H i l l}-\sqrt{\frac{2}{3}}\left(\sigma_{y}-R\right)=\sigma_{H i l l}-\sqrt{\frac{2}{3}}\left(\sigma_{y}+Q\left(1-e^{-\beta \kappa}\right)\right)
$$

where the expression for the equivalent stress $\sigma_{H i l l}$ is given as

$$
\begin{aligned}
\sigma_{\text {Hill }} & =\left(m_{1} \mathbf{Y}^{D} \cdot\left(\mathbf{Y}^{D}\right)^{T}+l_{4}\left(\frac{\operatorname{tr}\left(\mathbf{Y}^{D} \mathbf{C}_{p} \mathbf{M}_{\mathrm{ref}, 1}\right)}{\mathbf{C}_{p} \cdot \mathbf{M}_{\mathrm{ref}, 1}}\right)^{2}+m_{3}\left(\frac{\operatorname{tr}\left(\mathbf{Y}^{D} \mathbf{C}_{p} \mathbf{M}_{\mathrm{ref}, 2}\right)}{\mathbf{C}_{p} \cdot \mathbf{M}_{\mathrm{ref}, 2}}\right)^{2}\right. \\
& +2 l_{5} \frac{\operatorname{tr}\left(\mathbf{Y}^{D} \mathbf{Y}^{D} \mathbf{C}_{p} \mathbf{M}_{\mathrm{ref}, 1}\right)}{\mathbf{C}_{p} \cdot \mathbf{M}_{\mathrm{ref}, 1}}+2 l_{6} \frac{\operatorname{tr}\left(\mathbf{Y}^{D} \mathbf{Y}^{D} \mathbf{C}_{p} \mathbf{M}_{\mathrm{ref}, 2}\right)}{\mathbf{C}_{p} \cdot \mathbf{M}_{\mathrm{ref}, 2}} \\
& \left.+2 m_{2} \frac{\operatorname{tr}\left(\mathbf{Y}^{D} \mathbf{C}_{p} \mathbf{M}_{\mathrm{ref}, 1}\right)}{\mathbf{C}_{p} \cdot \mathbf{M}_{\mathrm{ref}, 1}} \frac{\operatorname{tr}\left(\mathbf{Y}^{D} \mathbf{C}_{p} \mathbf{M}_{\mathrm{ref}, 2}\right)}{\mathbf{C}_{p} \cdot \mathbf{M}_{\mathrm{ref}, 2}}\right)^{\frac{1}{2}}
\end{aligned}
$$

The plastic anisotropy parameters $m_{1}, m_{2}, m_{3}, l_{1}, l_{2}, l_{3}$ can be set in relation to the classical Hill parameters $F, G, H, L, M, N$.

Elastic anisotropy is described by the above mentioned dependence of $\psi_{e}$ on the structure tensors. The resulting relation for the second Piola-Kirchhoff stress tensor $\mathbf{S}$ is obtained by differentiation according to (7), i.e.

$$
\mathbf{S}=2 \mathbf{F}_{p}^{-1} \frac{\partial \psi_{e}}{\partial \mathbf{C}_{e}} \mathbf{F}_{p}^{-T}=2 \mathbf{F}_{p}^{-1} \frac{\partial\left(\psi_{e_{i s}}+\psi_{e_{a n}}\right)}{\partial \mathbf{C}_{e}} \mathbf{F}_{p}^{-T}=\mathbf{S}_{i s}+\mathbf{S}_{a n}
$$

where

$$
\mathbf{S}=\mu\left(\mathbf{C}_{p}^{-1}-\mathbf{C}^{-1}\right)+\frac{\Lambda}{2}\left(\operatorname{det} \mathbf{C}\left(\operatorname{det} \mathbf{C}_{p}\right)^{-1}-1\right) \mathbf{C}^{-1}
$$


and

$$
\begin{aligned}
\mathbf{S}_{a n} & =2 \gamma_{1} K_{1}\left(\frac{\operatorname{tr}\left(\mathbf{C} \mathbf{M}_{\mathrm{ref}, 1}\right)}{\mathbf{C}_{p} \cdot \mathbf{M}_{\mathrm{ref}, 1}}-1\right)^{\gamma_{1}-1} \frac{\mathbf{M}_{\mathrm{ref}, 1}}{\mathbf{C}_{p} \cdot \mathbf{M}_{\mathrm{ref}, 1}} \\
& +2 \gamma_{2} K_{2}\left(\frac{\operatorname{tr}\left(\mathbf{C} \mathbf{M}_{\mathrm{ref}, 2}\right)}{\mathbf{C}_{p} \cdot \mathbf{M}_{\mathrm{ref}, 2}}-1\right)^{\gamma_{2}-1} \frac{\mathbf{M}_{\mathrm{ref}, 2}}{\mathbf{C}_{p} \cdot \mathbf{M}_{\mathrm{ref}, 2}}
\end{aligned}
$$

$K_{1}, K_{2}, \gamma_{1}, \gamma_{2}$ are material constants describing the elastic anisotropy. It should be noted that due to the fact that both the Helmholtz free energy $\psi$ and the yield potential $\Phi$ depend on the non-constant structure tensors $\mathbf{M}_{1}$ and $\mathbf{M}_{2}$, which live in the intermediate configuration, we end up with a model for evolving elastic and plastic anisotropy. From (3) it is obvious that the larger the plastic deformation, the larger the deviation of the anisotropy axes in the intermediate configuration with respect to the anisotropy axes in the reference configuration.

\section{RESULTS AND DISCUSSION}

The proposed constitutive framework of finite strain elastoplasticity with evolving elastic and plastic anisotropy and combined hardening is implemented as a user material subroutine UMAT into the commercial finite element software package ABAQUS/Standard. In this section, we investigate the performance of the new material model by means of two numerical examples: deep drawing of a cylindrical cup and thermoforming of polymer blends. The results are compared to the results obtained with our recently published model for constant Hill-type plastic anisotropy [11].

\subsection{Cylindrical cup drawing}

The first example discusses the application of the material model to the simulation of the cylindrical cup drawing process to study the earing profile after forming. Deep drawing is an important process including a die, a blankholder, and a punch. The blank is mounted between the die and the blankholder and is formed into the die by means of the downwards moving punch. The specific dimensions of the tools and the process parameters are chosen according to [12].
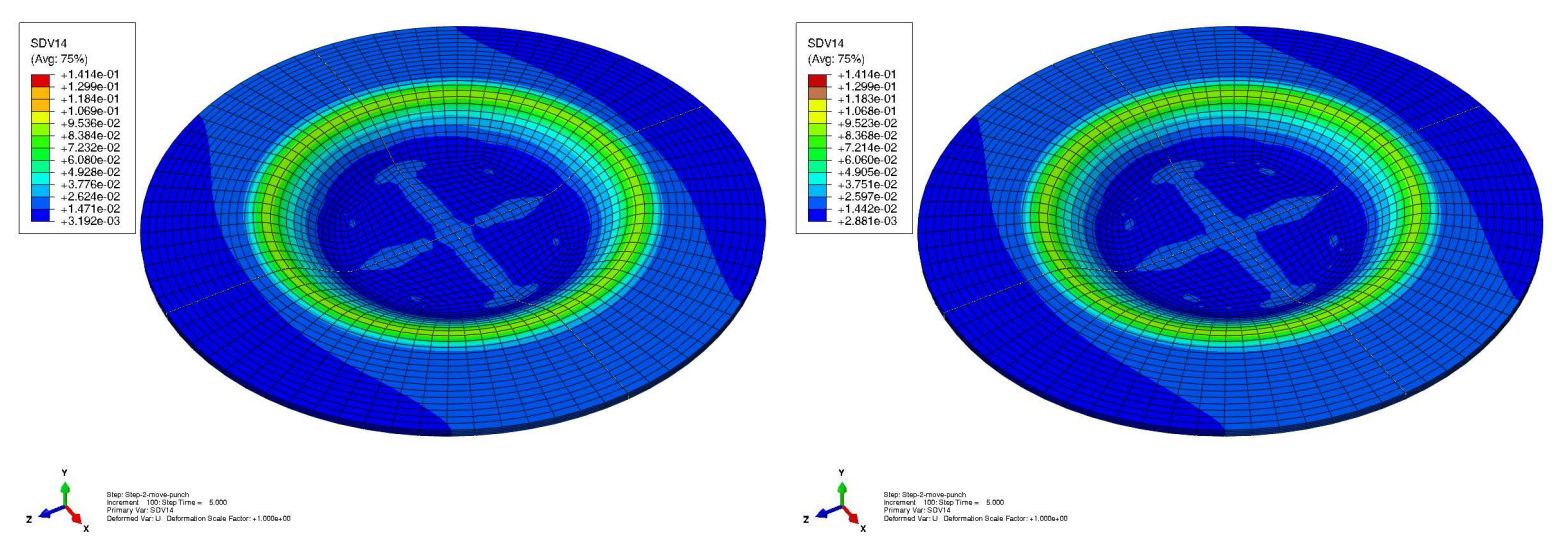

Figure 1: Accumulated plastic strain, step 1: (left) present model, (right) model of [11] 

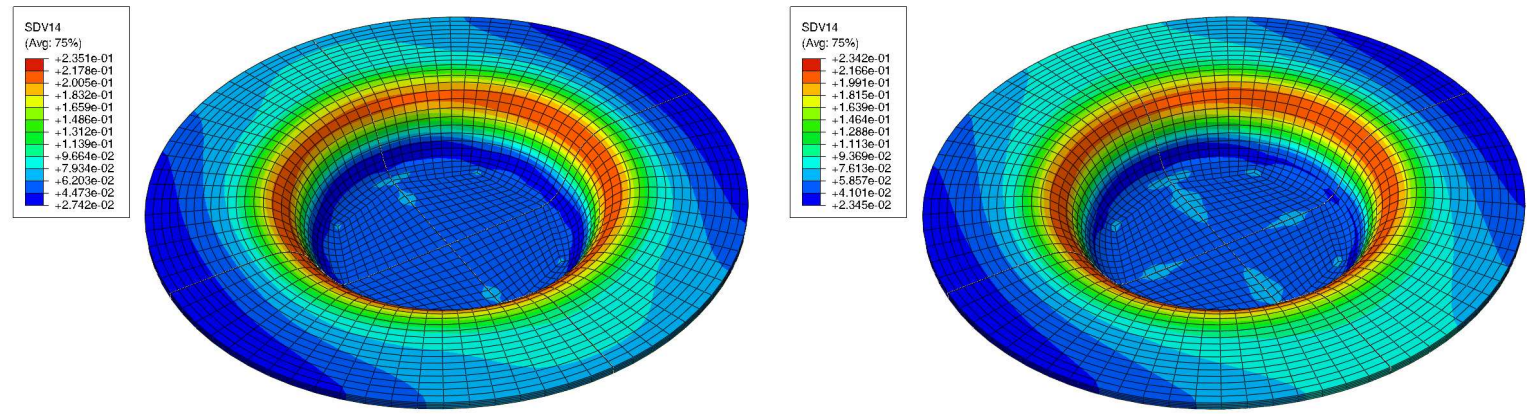

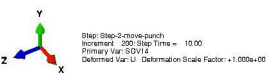

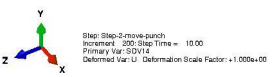

Figure 2: Accumulated plastic strain, step 2: (left) present model, (right) model of [11]
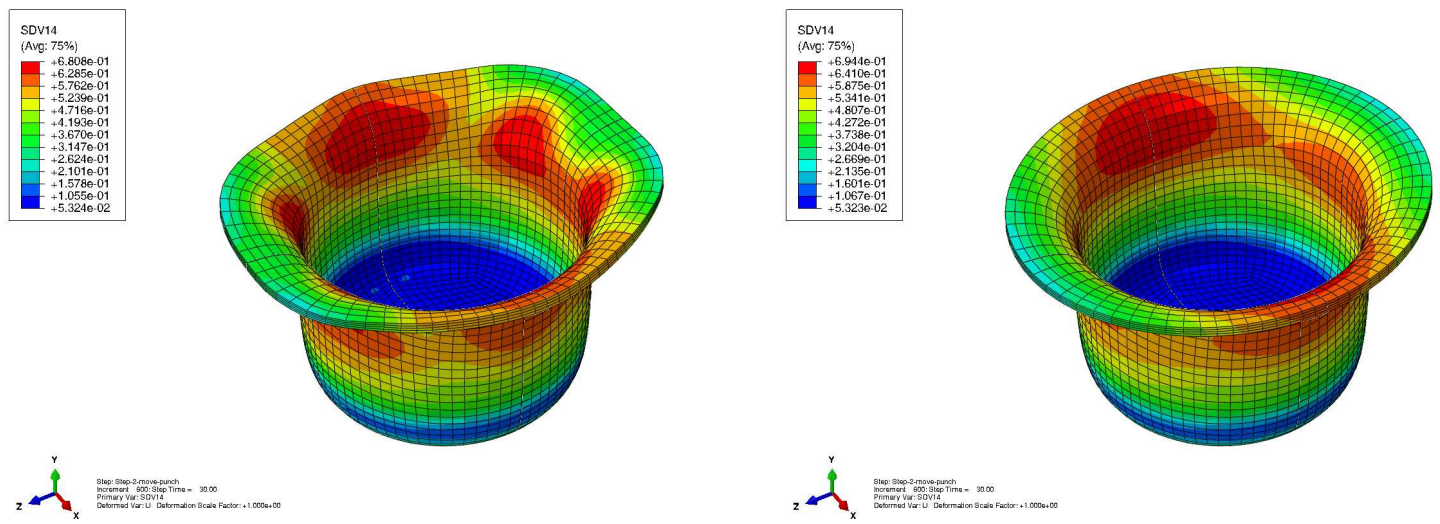

Figure 3: Accumulated plastic strain, step 3: (left) present model, (right) model of [11]
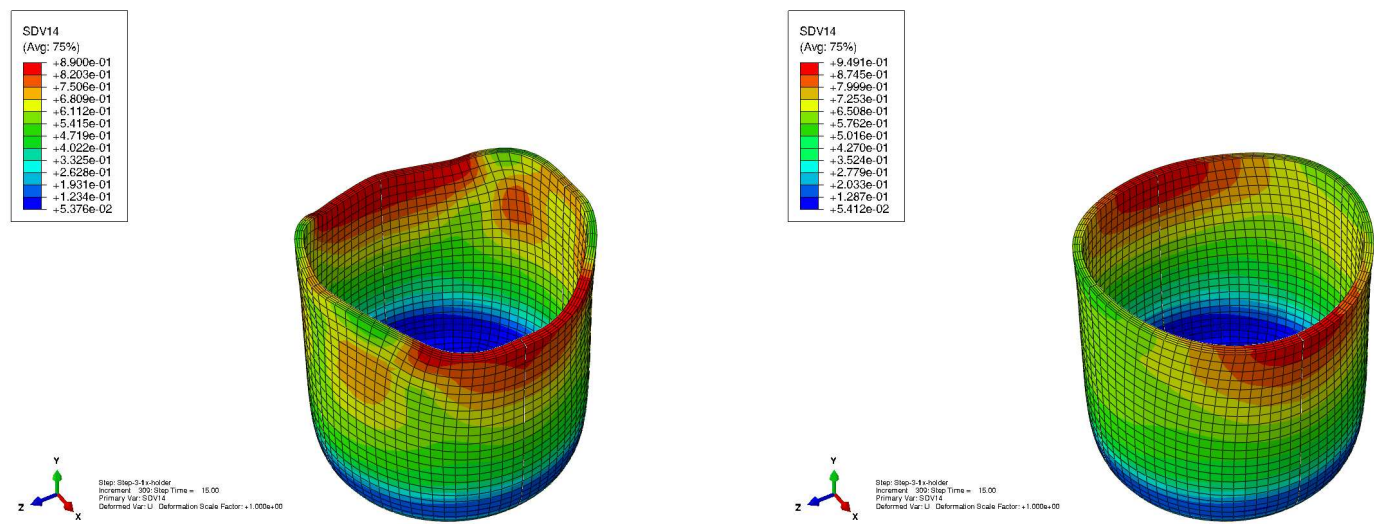

Figure 4: Accumulated plastic strain, step 4: (left) present model, (right) model of [11]

Figures 1 to 4 show the distribution of accumulated plastic strain at different stages of deep drawing, as computed by both models. No elastic anisotropy is considered in the simulations. The values of the material parameters for both models are identical: $\mu=80769$ $\mathrm{MPa}, \Lambda=121154 \mathrm{MPa}, \sigma_{y}=155 \mathrm{MPa}, Q=202 \mathrm{MPa}, \beta=15.5, F=0.3, G=0.7$, 
$H=0.5, L=M=N=1.5$. Thus, the only reason for the occurrence of different results should be the fact that the new model allows evolution of plastic anisotropy.

As expected, in the beginning the two simulation results are identical (Fig. 1). The reason for this is the fact that the description of initial plastic anisotropy is in both models based on a Hill-type orthotropy. With the increase of plastic deformation, the results commence to deviate (Fig. 1) and, quite interestingly, different number of ears is predicted (Figures 3 and 4). Obviously, for this set of parameters of plastic anisotropy ( $F=0.3, G=0.7$ and $H$ intermediate of them) the classical Hill result would be 2 ears. This result has been already stated in Hill's original paper ([2]). The present model, however, is capable of predicting eventually 6 ears (Fig. 4). This is a new result which, according to the authors' best knowledge, has not yet been presented in the literature by using a quadratic yield function.

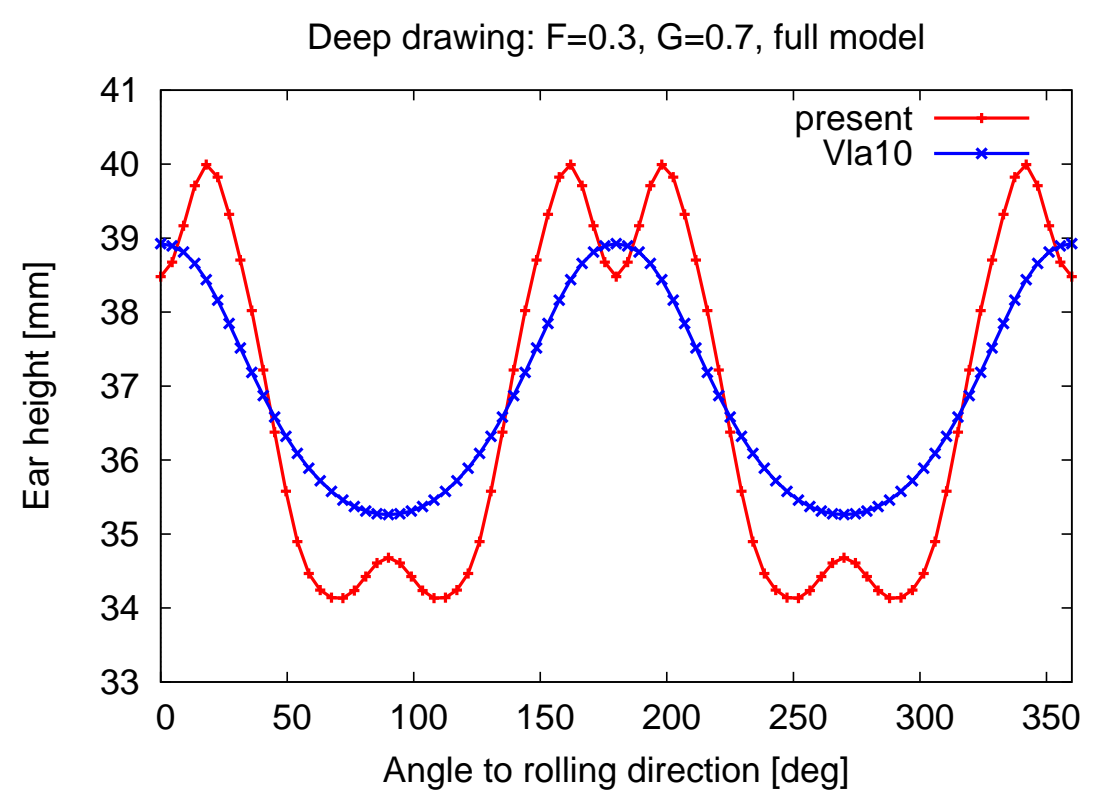

Figure 5: Cylindrical cup drawing, earing distribution: present model and model of [11], i.e. (Vla10)

Fig. 5 illustrates the simulated earing profiles of the cups obtained by the two models. The new model predicts stronger anisotropy (higher ears, lower valleys) and 6 ears instead of 2. Obviously, evolution of plastic anisotropy together with a rotation of the anisotropy axes take place.

Fig. 6 shows the punch force-displacement curves for the two anisotropic models. Due to the fact that for small plastic deformations the structure tensors in the intermediate configuration do not differ much from the structure tensors in the reference configuration, the anisotropic force-displacement curves remain almost identical up to a displacement of approximately $20 \mathrm{~mm}$. Beyond that, plastic deformation is considerable and the two curves begin to deviate significantly. 


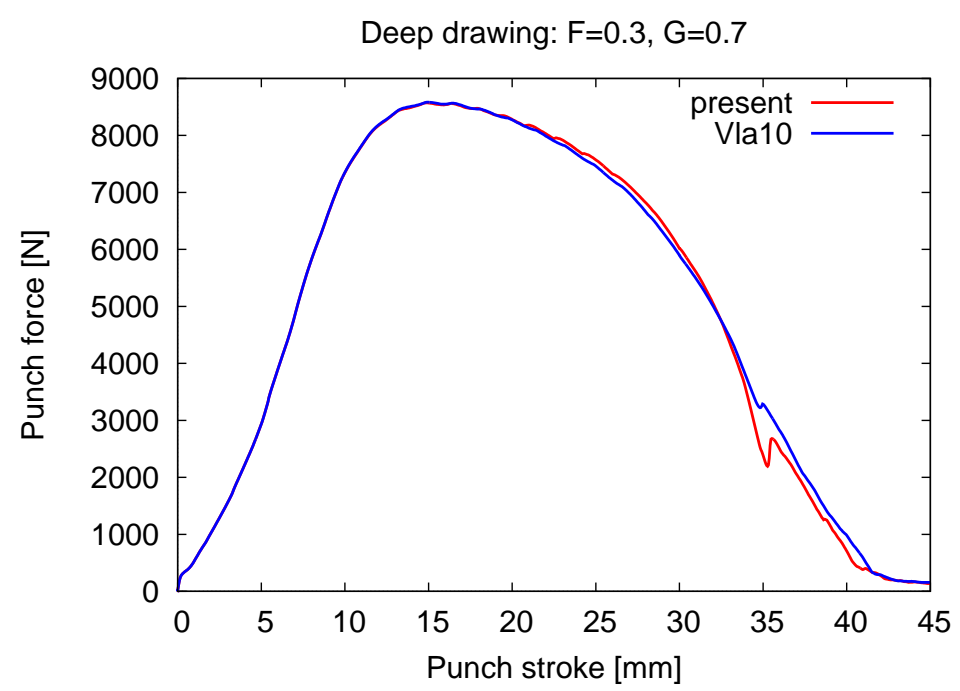

Figure 6: Cylindrical cup drawing, punch force-displacement curves: present model and model of [11], i.e. (Vla10)

\subsection{Thermoforming}

The second example deals with the simulation of the process of thermoforming of TPO (Thermo-Plastic Olefin) sheets. TPO polymer/filler blends are used extensively in the automotive industry. The process of thermoforming is one of the oldest and most common methods of processing plastic materials. It represents a reshaping of a thermoplastic forming material onto moulded tools at its own forming temperature. Basically, such a process consists of inserting a thermoplastic sheet in a cold state into the forming clamp area, pre-heating it to the desired temperature, and then moving the mould upwards to form the sheet. The trapped air is sucked out with the help of a vacuum system and once cooled a reverse air supply is activated to remove the plastic part from the mould.

The motivation for investigating this application into the present study comes from the fact that thermoforming involves large elastic strains (up to $15 \%$, see [10]). The assumption of small elastic strains usually made in metal plasticity is therefore no longer justified. Thus we see here a very interesting example for a situation where large elastic strains have to be incorporated into the modelling approach. Furthermore, considering the thermoforming simulation of a material with large elastic strains allows us to study the influence of elastic anisotropy on the results.

Figures 7, 8 and 9 show the stress-strain curves for a TPO blend, obtained at orientations of 0,45 and 90 degrees with respect to the rolling direction. The elasticity and hardening parameters are taken from [10] and read: $\mu=2 \mathrm{MPa}, \Lambda=198.7 \mathrm{MPa}, \sigma_{y}=0.6 \mathrm{MPa}, H=1$. $\mathrm{MPa}$ (linear isotropic hardening, i.e. $R=-H \kappa$ ), $b=0.001, c=0.6 \mathrm{MPa}$.

Fig. 7 depicts the stress-strain curves for an elastically anisotropic $\left(K_{1}=1 \mathrm{MPa}\right.$, $\left.K_{2}=3 \mathrm{MPa}, \gamma_{1}=\gamma_{2}=1\right)$ but plastically isotropic $(F=G=H=0.5, L=M=N=1.5$ ) material. Analogously, Fig. 8 shows the stress-strain behaviour of an elastically isotropic $\left(K_{1}=2 \mathrm{MPa}, K_{2}=2 \mathrm{MPa}, \gamma_{1}=\gamma_{2}=1\right)$ but plastically isotropic $(F=0.534, G=0.634$, $H=0.418, L=M=1.5$ and $N=2.05)$ material. Finally, combining both elastic and 


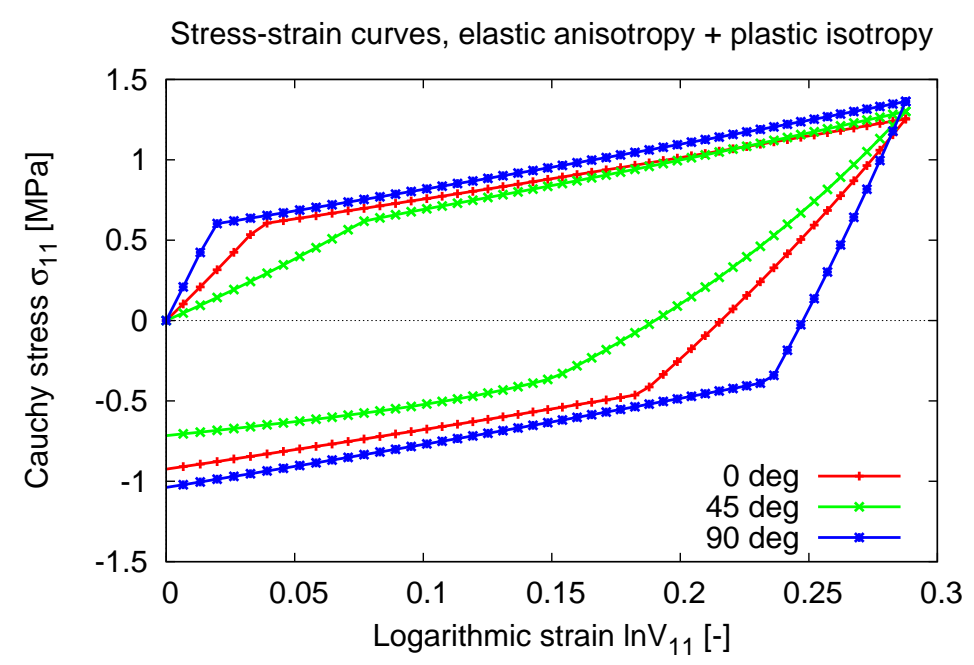

Figure 7: Uniaxial tension, elastic anisotropy and plastic isotropy: 0, 45 and 90 degrees to rolling direction

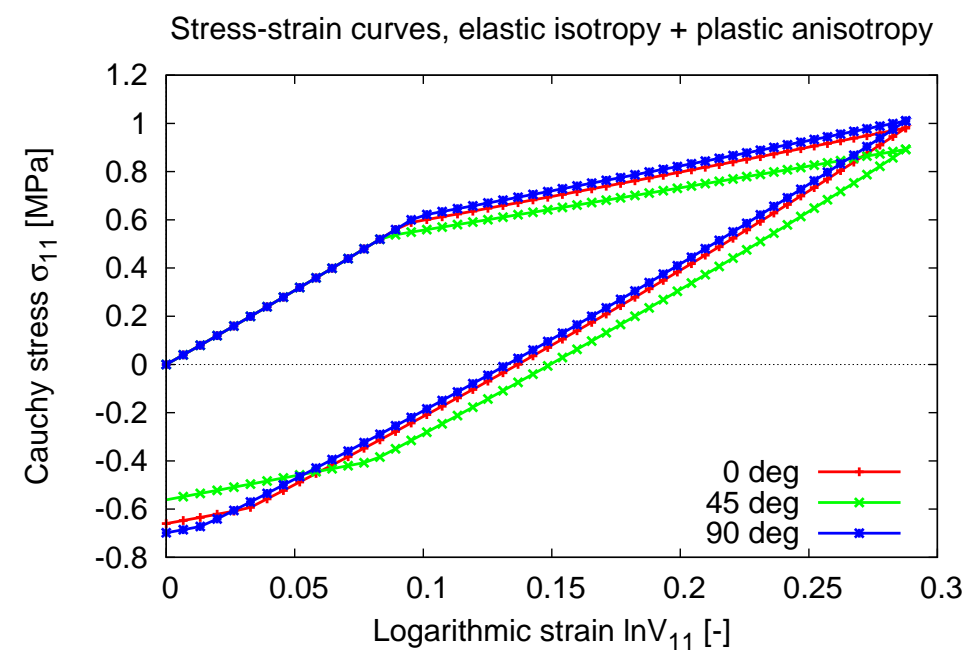

Figure 8: Uniaxial tension, elastic isotropy and plastic anisotropy: 0, 45 and 90 degrees to rolling direction

plastic anisotropy and utilizing the same sets of material parameters as stated above results in the response shown in Fig. 9.

Fig. 10 shows the simulation of the thermoforming process, as performed in this research. A quarter of a circular thermoplastic sheet is clamped at the nodes along its perimeter and is placed into contact with an upwards moving rigid mould. After the mould reaches the prescribed forming distance, vacuum is simulated by applying a negative pressure on the top surface of the sheet. This results in the sheet sticking closely to the mould and obtaining its final shape (Fig. 10).

The FE simulation is again performed by means of ABAQUS/Standard. A fine mesh 


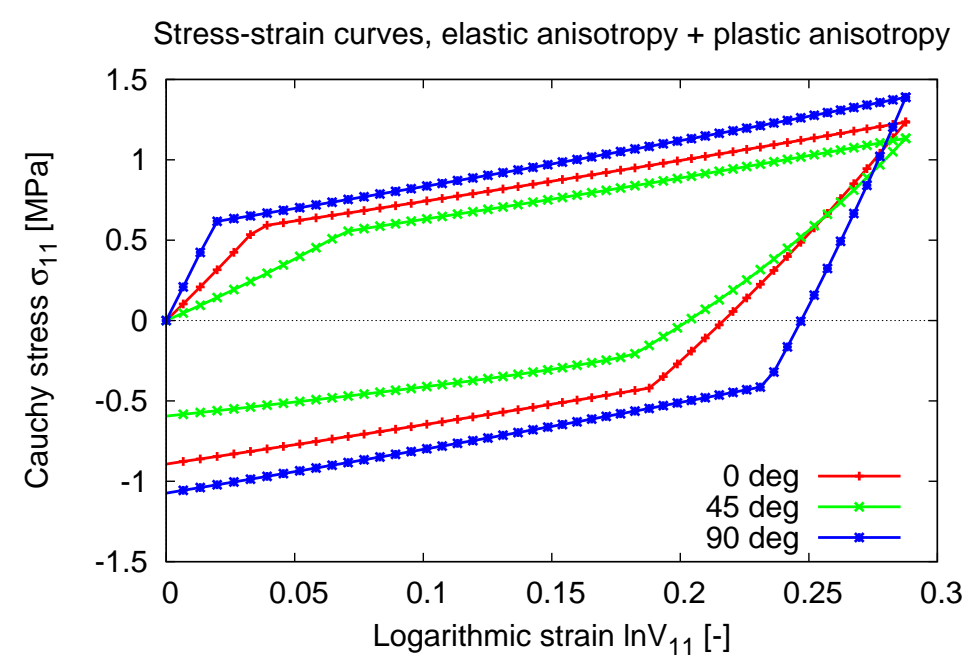

Figure 9: Uniaxial tension, elastic anisotropy and plastic anisotropy: 0, 45 and 90 degrees to rolling direction
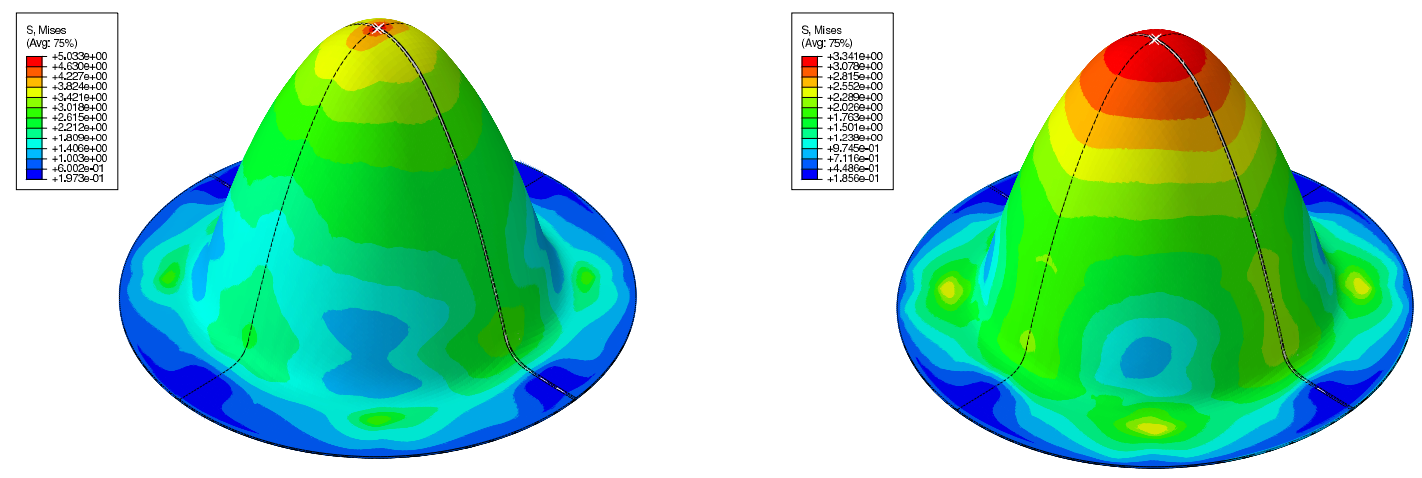

Figure 10: Thermoforming, distribution of von Mises stress: (left) elastic anisotropy and plastic anisotropy, (right) elastic isotropy and plastic anisotropy

of approximately 12000 (radius x circumference $\mathrm{x}$ thickness $=80 \times 126 \times 2$ ) C3D8R reduced integration continuum elements is used. It should be noted that due to the purely mechanical material model formulation, no temperature influences are taken into account. The thermoforming simulation is performed by utilizing the three sets of anisotropic material parameters (i.e., elastically anisotropic - plastically isotropic, elastically isotropic - plastically anisotropic, and elastically anisotropic - plastically anisotropic). In addition, a complete isotropic simulation with neither elastic nor plastic anisotropic properties is added for comparison purpose. The computed force-displacement curves for the rigid mould are illustrated in Fig. 11. Obviously, elastic anisotropy plays an important role in the simulation. The calculated force is generally higher for the materials with elastic anisotropy due to the fact that the amount of the anisotropic elastic Helmholtz strain energy function $\psi_{e_{a n}}$ is added to the isotropic one, yielding an additional stress $\mathbf{S}_{a n}$. 


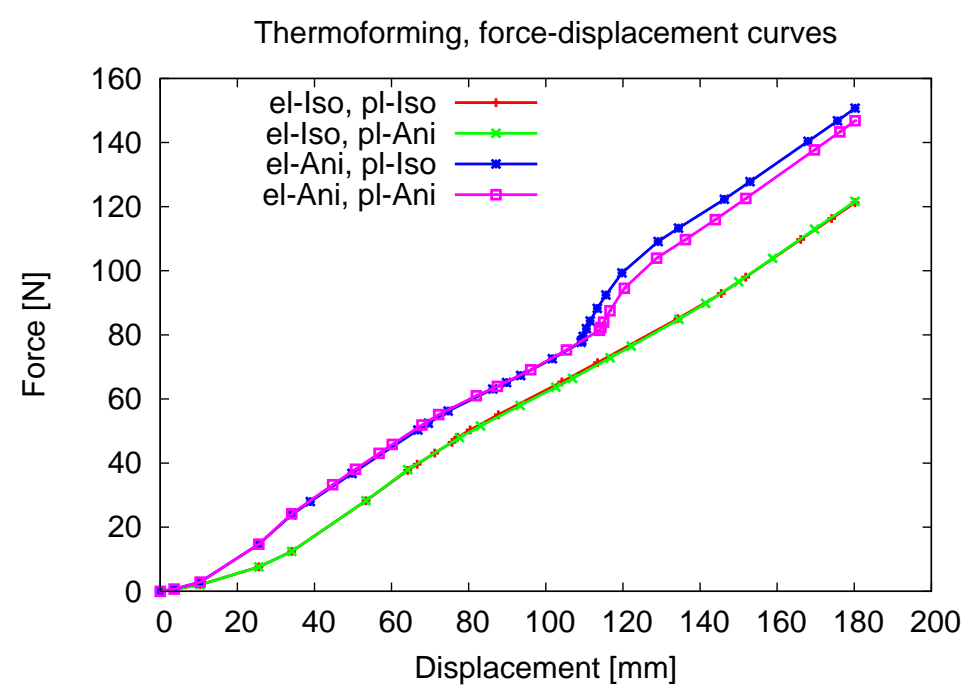

Figure 11: Thermoforming, punch force-displacement curves

\section{CONCLUSIONS}

A finite strain material model for evolving elastic and plastic anisotropy and combined hardening has been presented. The numerical results show that more pronounced anisotropy can be predicted, as compared to the results obtained by a Hill-type plastic orthotropy. Interestingly, the new model is capable of simulating rotation of the preferred material orientations and predicting 6 ears. Clearly, experimental evidence of evolving elastic and plastic anisotropy is required in order to be able to tune the model and investigate its capabilities of realistically describing material anisotropies.

\section{References}

[1] Barlat, F., Yoon, J. W., Cazacu, O.; On linear transformations of stress tensors for the description of plastic anisotropy, International Journal of Plasticity 23:876-896, 2007.

[2] Hill, R.; A theory of the yielding and plastic flow of anisotropic metals, Proceedings of the Royal Society of London A193:281-297, 1948.

[3] Li, S., Hoferlin, E., Van Bael, A., Van Houtte, P., Teodosiu, C.; Finite element modeling of plastic anisotropy induced by texture and strain-path change, International Journal of Plasticity 19:647-674, 2003.

[4] Miehe, C., Apel, N., Lambrecht, M.; Anisotropic additive plasticity in the logarithmic strain space: modular kinematic formulation and implementation based on incremental minimization principles for standard materials, Computer Methods in Applied Mechanics and Engineering 191:5383-5425, 2002.

[5] Papadopoulos, P., Lu, J.; On the formulation and numerical solution of problems in anisotropic finite plasticity, Computer Methods in Applied Mechanics and Engineering 190:4889-4910, 2001.

[6] Reese, S.; Meso-macro modelling of fibre-reinforced rubber-like composites exhibiting large elastoplastic deformation, International Journal of Solids and Structures 40:951-980, 2003.

[7] Sansour, C., Karsaj, I., Soric, J.; On a numerical implementation of a formulation of anisotropic continuum elastoplasticity at finite strains, Journal of Computational Physics 28:732-742, 2008. 
[8] Stoughton, T., Yoon, J. W.; Anisotropic hardening and non-associated ow in proportional loading of sheet metals, International Journal of Plasticity 25:1777-1817, 2009.

[9] Vladimirov, I. N., Pietryga, M. P., Reese, S.; On the modeling of nonlinear kinematic hardening at finite strains with application to springback - Comparison of time integration algorithms, International Journal for Numerical Methods in Engineering 75:1-28, 2008.

[10] Vladimirov, I. N., Pietryga, M. P., Reese, S.; Prediction of springback in sheet forming by a new finite strain model with nonlinear kinematic and isotropic hardening, Journal of Materials Processing Technology 209:4062-4075, 2009.

[11] Vladimirov, I. N., Pietryga, M. P., Reese, S.; Anisotropic finite elastoplasticity with nonlinear kinematic and isotropic hardening and application to sheet metal forming, International Journal of Plasticity 26:659-687, 2010.

[12] Kishor, N., Kumar, D. R.; Optimization of initial blank shape to minimize earing in deep drawing using nite element method, Journal of Materials Processing Technology 130-131:20-30, 2002. 